\title{
Outcomes, Microbiology and Antimicrobial Usage in Pressure Ulcer-Related Pelvic Osteomyelitis: Messages for Clinical Practice
}

\author{
Clark D. Russell1,2*, Shao-Ting Jerry Tsang $2,3^{*}$, Alasdair Hamish R. W. Simpson ${ }^{3 \#, ~ R e b e c c a ~ K . ~ S u t h e r l a n d ~}{ }^{1 \boxplus}$ \\ 1. NHS Lothian Infection Service, Regional Infectious Diseases Unit, Western General Hospital, Edinburgh, U.K. \\ 2. University of Edinburgh Centre for Inflammation Research, Queen's Medical Research Institute, Edinburgh BioQuarter, Edinburgh, U.K. \\ 3. Department of Orthopaedic Surgery, University of Edinburgh, Chancellor's Building, Edinburgh, U.K.
}

${ }^{*} \mathrm{CDR}$ and STJT contributed equally

\#Joint senior authors

$\square$ Corresponding author: Dr. Rebecca K. Sutherland, Regional Infectious Diseases Unit, Western General Hospital, Crewe Road South, Edinburgh EH4 2XU, U.K. E-mail: Rebecca.Sutherland@nhslothian.scot.nhs.uk

(๑) The author(s). This is an open access article distributed under the terms of the Creative Commons Attribution License (https://creativecommons.org/licenses/by/4.0/). See http://ivyspring.com/terms for full terms and conditions.

Received: 2019.11.04; Accepted: 2019.12.27; Published: 2020.03.26

\begin{abstract}
Introduction: Pressure ulcer-related pelvic osteomyelitis is a relatively under-studied entity in the field of bone infection. We sought to add to the limited evidence base for managing this challenging syndrome.

Methods: Cases were identified retrospectively from a surgical database and hospital discharge codes at a U.K. tertiary centre (2009-2018). Risk factors associated with outcomes were analysed by logistic regression.

Results: We identified 35 patients (mean age 57.4 years), 69\% managed with a combined medical and surgical approach, with mean follow-up of 3.7 years from index admission. Treatment failure (requiring further surgery or intravenous antimicrobials) occurred in $71 \%$ and eventual ulcer healing in $36 \%$. One-year mortality was $23 \%$. Lack of formal care support on discharge, post-traumatic (asensate) neurological deficit and index CRP $(>184 \mathrm{mg} / \mathrm{L})$ were associated with treatment failure $(p=0.001)$. Age $(>59.5$ years), lack of attempted soft tissue coverage, haemoglobin $(<11 \mathrm{lg} / \mathrm{L})$ and albumin $(<25 \mathrm{~g} / \mathrm{L})$ were associated with non-healing ulcers $(p=0.003)$. Superficial wound swabs had low sensitivity and specificity compared to deep bone microbiology. Infection (based on deep bone microbiology from 46 infection episodes) was usually polymicrobial (87\%), commonly involving S. aureus, Enterococci, GNB and anaerobes. Antimicrobial duration ranged from 0-103 days (mean 54) and was not associated with subsequent treatment failure.

Conclusions: Attempted soft tissue coverage after surgical debridement, ensuring appropriate support for personal care after discharge and nutritional optimisation could improve outcomes. Superficial wound swabs are uninformative and deep bone sampling should be pursued. Long antimicrobial courses do not improve outcomes. Clinicians should engage patients in anticipatory care planning.
\end{abstract}

Key words: osteomyelitis, pressure ulcer, pelvis, spinal cord injuries

\section{Introduction}

Pelvic osteomyelitis occurring as a complication of pressure ulcers results in substantial morbidity and therapeutic challenges, but remains a relatively under-studied entity in the field of bone infection. Such pressure ulcers arise due to immobility, usually in the context of neurological dysfunction [1]. Concomitant urinary incontinence can lead to skin irritation further compromising barrier function, and faecal incontinence can lead to wound soiling and introduction of enteric bacteria. Bone sequestra and 
necrotic connective tissue, devoid of a blood supply, provide a persistent nidus of infection and impair local antimicrobial penetration following systemic administration. Wound closure and healing following surgical intervention are at high risk of failure [2].

A recent systematic review of this challenging syndrome provides an overview of the limited available data pertaining to diagnosis and management [3]. Diagnosis itself can be difficult, and contrary to the widely held belief, exposed bone is not necessarily synonymous with histological evidence of osteomyelitis [3]. Furthermore, the specificity of CT and MRI has been questioned when histology is considered the gold standard [4-9]. The optimum duration of antimicrobial therapy has not been defined, nor the sub-group of patients who will derive benefit from surgical intervention.

Utilising a single centre cohort of patients we sought to add to the limited evidence base for this challenging syndrome. We aimed to ask clinically-relevant questions including what factors are associated with treatment failure and failure of ulcer healing, how do deep and superficial microbiological samples compare, and whether antimicrobial duration is associated with outcome?

\section{Methods}

\section{Data collection}

Patients from a U.K. tertiary centre were identified from a local database of patients undergoing surgical management of osteomyelitis, supplemented by an electronic search of the electronic patient record (TrakCare $\left.{ }^{\circledR}\right)$ for episodes with diagnoses coded (Table S1) as osteomyelitis of the pelvis, sacrum, coccyx or ischium, or infected pressure ulcers (December 2009-December 2018). Cases identified by the latter search were then reviewed to determine if they met the case definition (below). The case log was created following a search of the pan-regional electronic perioperative management system (Operating Room Scheduling and Office System), reflecting an entire regional surgical practice. TrakCare ${ }^{\circledR}$ is a region-wide electronic medical record system. It collects the medical data from patient interactions in all secondary and tertiary centres throughout the region. Death is recorded on TrakCare ${ }^{\circledR}$ and linked to national death records by the patient's community health index number, a unique patient identifier assigned to all users of the healthcare system in Scotland. Chart review was undertaken and relevant clinical, radiological and microbiological details were recorded. The project was reviewed and approved by the NHS Lothian MusculoskeletalOrthopaedic Quality Improvement Team.

\section{Definitions}

Cases were defined as adults ( $\geq 18$ years) requiring inpatient management of clinically and radiologically diagnosed pressure ulcer-related pelvic osteomyelitis. Patients were excluded if they presented with soft tissue infection involving an ulcer or visible bone (i.e. grade 4 ulcer) without evidence of underlying osteomyelitis. The index episode was defined as the initial presentation requiring inpatient management including the first surgical procedure. Treatment failure was defined as: (i) further surgical debridement, (ii) further positive bone cultures, or (iii) re-admission for intravenous antimicrobials for treatment of pelvic osteomyelitis. This expands on a recently used definition with the addition of requirement for further antimicrobials, which we contend is a relevant outcome for patients [10]. Patients requiring surgical debridement underwent wide resection with dissection down to bleeding bone. Bone prominences were removed to prevent future skin pressure. Where possible, defects were closed primarily or covered with a flap reconstruction. If neither option was possible in the first instance, negative pressure wound dressings were applied to facilitate delayed closure +/- soft tissue reconstruction. Multiple intraoperative deep bone samples were taken for microbiological investigation (usually six) and at least one sample for histological analysis (soft tissue $+/$ - bone).

\section{Significant microbiology}

Isolates of uncertain clinical significance in the context of osteomyelitis (e.g. Corynebacterium spp., coagulase-negative Staphylococci [excluding $S$. lugdunensis], viridans group Streptococci, fungi) were considered as significant if they were recovered from $>1$ deep sample from the same procedure and had a similar antibiogram, or if they were recovered from the same patient during a subsequent infection episode.

\section{Statistical analysis}

Fisher's exact-test (or a Chi-square test where appropriate) was used to analyse the difference between groups for categorical variables, and a univariate analysis of variance (or Mann Witney $U$ test for non-parametric data) for continuous variables. A Kaplan Meier survival curve with a log-rank analysis was used for comparative evaluations of treatment failure and mortality in time. Possible risk factors for treatment failure were selected and analysed using univariate analysis as detailed above. Receiver operator characteristic (ROC) analysis was performed to quantitate the predictive performance of risk factors identified on univariate analysis, in 
addition the Youden's J statistic was calculated for identified continuous variables. Identified variables on univariate analysis were assessed for multicollinearity in a binomial logistic regression analysis with only statistically significant variables included in the risk score model. All analyses were two-tailed and p-values $<0.05$ were considered to be statistically significant. Statistical analysis was performed using SPSS, version 24.0 (SPSS Inc. Chicago, IL) and Prism, version 8.0 (GraphPad Software Inc. San Diego, CA).

\section{Results}

\section{Patient characteristics}

Thirty five patients with pressure ulcer-related pelvic osteomyelitis were included. The mean age was 57.4 years (95\% CI 52.1-62.8) and 26/35 (74\%) were male. Most patients (32/35) had neurological dysfunction resulting in immobility, with frailty $(2 / 35)$ and rheumatoid arthritis $(1 / 35)$ as predisposing factors in the remaining cases. Other co-morbidities were uncommon (median 0 , range 1-3; Table 1). The most common site of osteomyelitis was the ischium $(18 / 35)$. Full patient characteristics are reported in Table 1. 31/35 index admissions were directly due to pelvic osteomyelitis (due to other intercurrent infections in the remaining 4) and in 27 such instances the patient was acutely unwell due to osteomyelitis and/or skin and soft tissue infection (SSTI) involving the ulcer (admitted electively for surgery in remaining 4). The responsible pressure ulcers had been present for a median of 4 months (IQR 1.75-12.0) and were most commonly grade four $(21 / 35)$ or three $(13 / 35)$. There was one case of squamous cell carcinoma as a complication of the infection.

Diagnostic parameters at index presentation are presented in Table 1. Clinical signs of SSTI involving the ulcer were present in 29/35 cases. Samples of bone were sent to histopathology in nine cases, with active inflammation identified in seven. In the two cases where only reactive changes were reported, MRI identified sequestra and erosions, and bone samples from the same operations sent to microbiology were culture-positive.

\section{Management}

The majority of patients were managed with a combined medical and surgical approach (24/35). One patient underwent flap reconstruction during their index presentation followed by one re-admission for debridement and flap elevation. Three patients underwent flap reconstruction on their second re-admission and required no further intervention. Of the patients who underwent direct primary closure, 11/14 experienced wound breakdown with six requiring further surgical intervention and five requiring further antimicrobials only. They required a median of one further admission (range 0-7), with $7 / 14$ having wounds that had healed at the time of chart review.

The mean total antimicrobial duration during the index episode was 54 days (standard deviation \pm 31 days), but this was highly variable (Figure 1A), with a range of 0-103 days. There was no association between antimicrobial duration and subsequent treatment failure (51.0 days treatment success, 55.7 days treatment failure, $p=0.7$ ). When patients were stratified into receiving $\leq 6$ weeks or $>6$ weeks of antimicrobials there remained no association with subsequent treatment failure $(p=0.7)$. Patients received a median of 4 antimicrobials in total (IQR 26), with flucloxacillin, vancomycin, metronidazole, piperacillin-tazobactam and ciprofloxacin constituting over half (Figure 1B). Broad spectrum beta-lactams (piperacillin-tazobactam, co-amoxiclav, meropenem) were used for average durations of 17, 30 and 19 days respectively (Figure 1C). Despite the extensive antimicrobial exposure there was only one case of Clostridioides difficile infection. Seven patients developed adverse drug reactions involving antimicrobials, including nephrotoxicity (vancomycin and gentamicin), hyper-sensitivity (teicoplanin and co-trimoxazole) and anaemia (linezolid).

\section{Outcomes}

\section{Follow-up and mortality}

At time of chart review 15/35 (43\%) patients had died, with 8/35 (23\%) dying within twelve months of their index episode. Kaplan-Meier analysis provided an estimated median time to death from index episode of 7.0 years (95\% CI 2.7-11.3) for the entire cohort (Figure 2A). Median time to death in patients with healed pressure ulcers was 7.0 years $(95 \%$ CI not available) and unhealed pressure ulcers was 2.0 years (95\% CI 0.6-3.4 years; $\mathrm{p}=0.003$, Figure 2B). Excluding the 15 deaths, there was a mean follow-up of 3.7 years (range 1.3-7.2).

\section{Ulcer healing}

Unhealed pressure ulcers were present in 21/33 $(64 \%)$ patients at the time of chart review or death. Univariate analysis identified older age (mean 63.4 vs. 48.9 years, $\mathrm{p}=0.010)$, diagnosis of diabetes mellitus (7/21 vs. $0 / 12, p=0.032)$, lower haemoglobin and albumin at index presentation (mean $105 \mathrm{vs} .125 \mathrm{~g} \mathrm{~L}^{-1}$, $\mathrm{p}=0.007$; and 22.1 vs. $27.2 \mathrm{~g} \mathrm{~L}^{-1}, \mathrm{p}=0.040$, respectively), and not attempting soft tissue coverage at index presentation $(8 / 20$ vs. $10 / 12$ primary closure as opposed to no closure or negative pressure wound dressing, $p=0.028$ ) to be associated with an unhealed ulcer. Receiver operating characteristic (ROC) 
analysis is presented in Table 2. Binomial logistic regression analysis $\left(X^{2}(4)=15.781, p=0.003\right)$ found that admission haemoglobin $\left(<111 \mathrm{~g} \mathrm{~L}^{-1}\right)$, admission albumin $\left(<25 \mathrm{~g} \mathrm{~L}^{-1}\right)$, lack of soft tissue coverage, and patient age at index presentation ( $>59.5$ years) accounted for $79.3 \%$ (Nagelkerke $\mathrm{R}^{2}$ ) of the variance in the failure of pressure ulcers to heal and correctly classified $90.5 \%$ of cases (Table S2).

\section{Treatment failure}

Treatment failure occurred in 25/35 (71.4\%) cases. The median time from index presentation was 9.3 months (95\% CI 2.7-15.9) (Figure 2C). Univariate analysis found that lack of formal care support on discharge (relative risk ratio [RRR] 3.2, $\mathrm{p}=0.027$ ), an underlying asensate post-traumatic neurological deficit (RRR N/A, p=0.030), and higher index admission C-reactive protein (194 vs. $105 \mathrm{mg} \mathrm{L}^{-1}$, $\mathrm{p}=0.021$ ) (Figure 2D) were associated with treatment failure. Bacteraemia on index admission was not associated with subsequent treatment failure $(p=1.0)$. Receiver operating characteristic (ROC) analysis is presented in Table 2. Binomial logistic regression analysis $\left(X^{2}(3)=15.588, p=0.001\right)$ found that index CRP (>184mg L-1), lack of formal care support and a post-traumatic neurological deficit accounted for $52.4 \%$ (Nagelkerke $\mathrm{R}^{2}$ ) of the variance in treatment failure and correctly classified $82 \%$ of cases (Table S2).

b

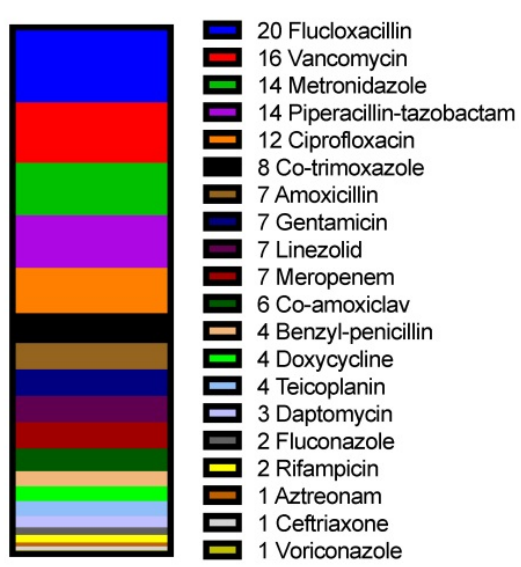

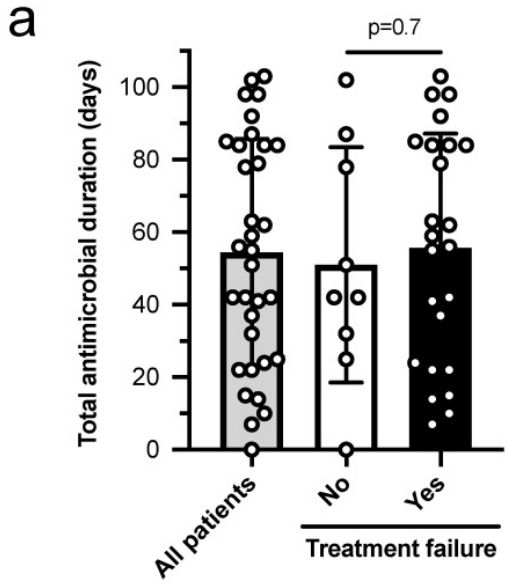

C

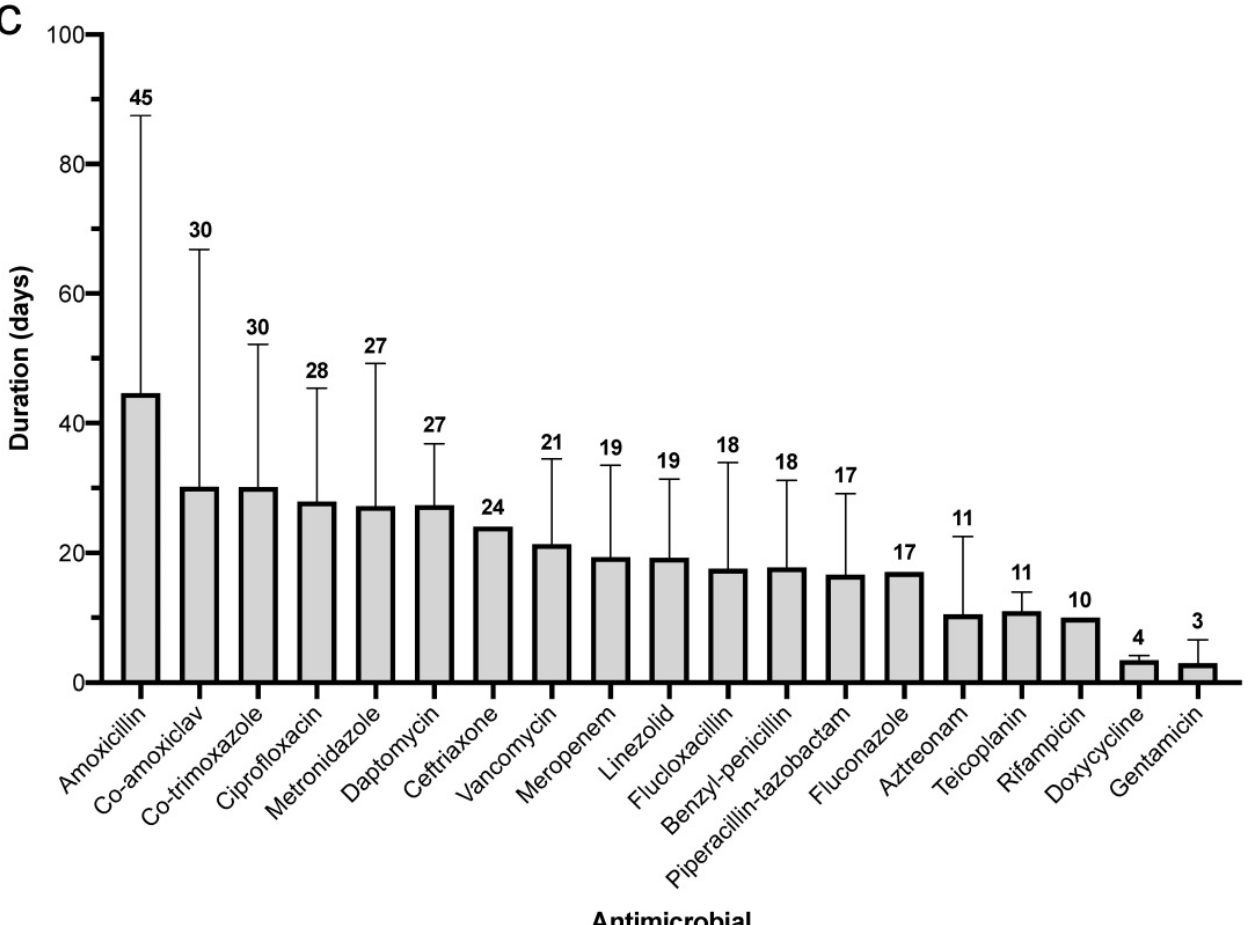

Figure 1. Antimicrobial usage. a) The total antimicrobial duration at index presentation was not associated with subsequent treatment failure (groups compared by unpaired $t$ test). b) Antimicrobial agents used during management of index episode. c) Duration of each antimicrobial agent during index episodes (number is mean). Antimicrobial data available for 33 patients. Graphs show mean and standard deviation. 


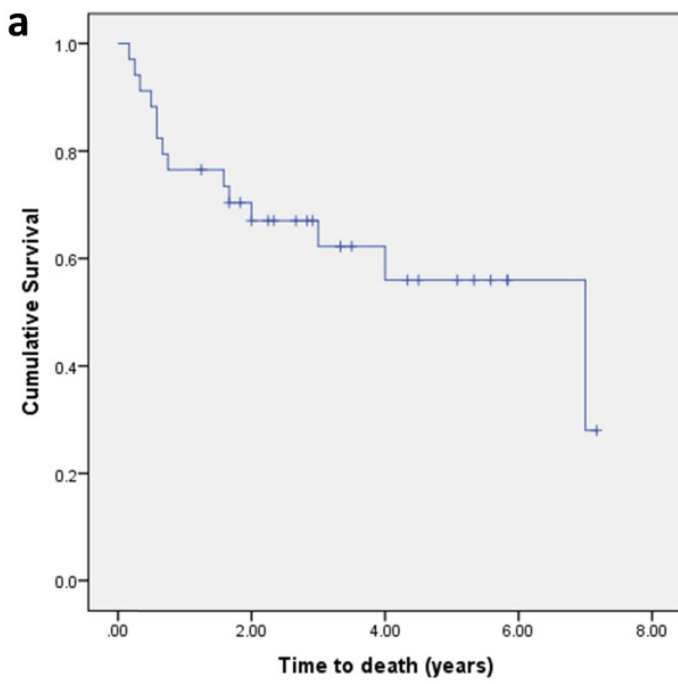

C

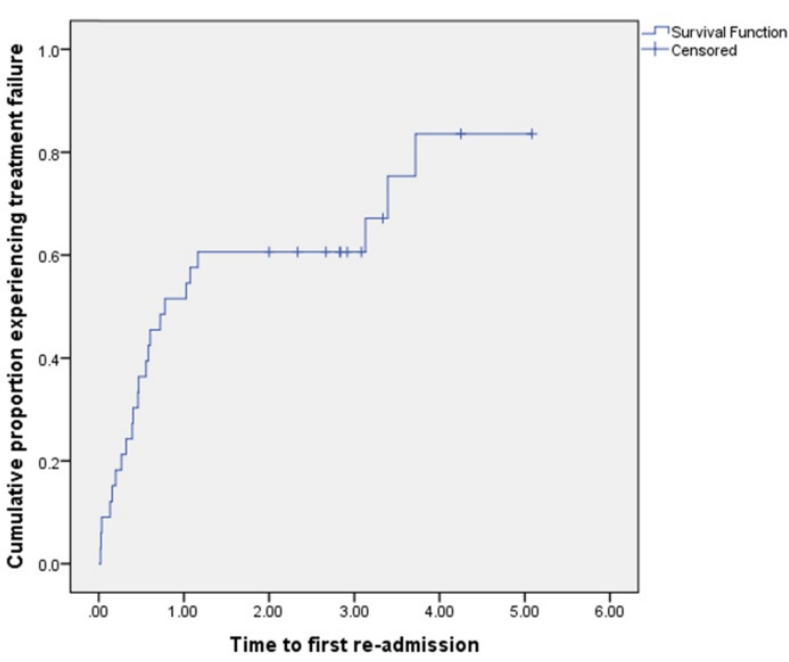

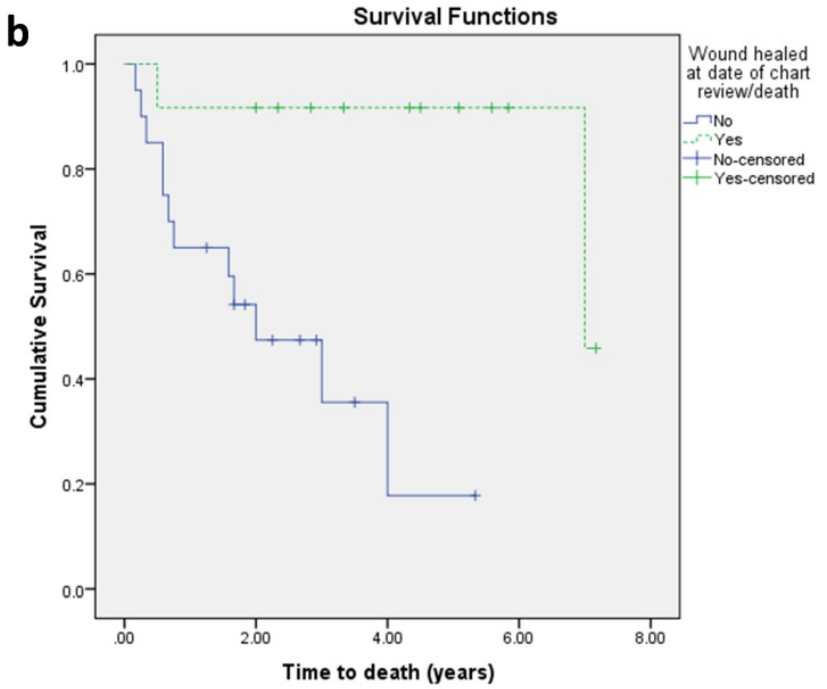

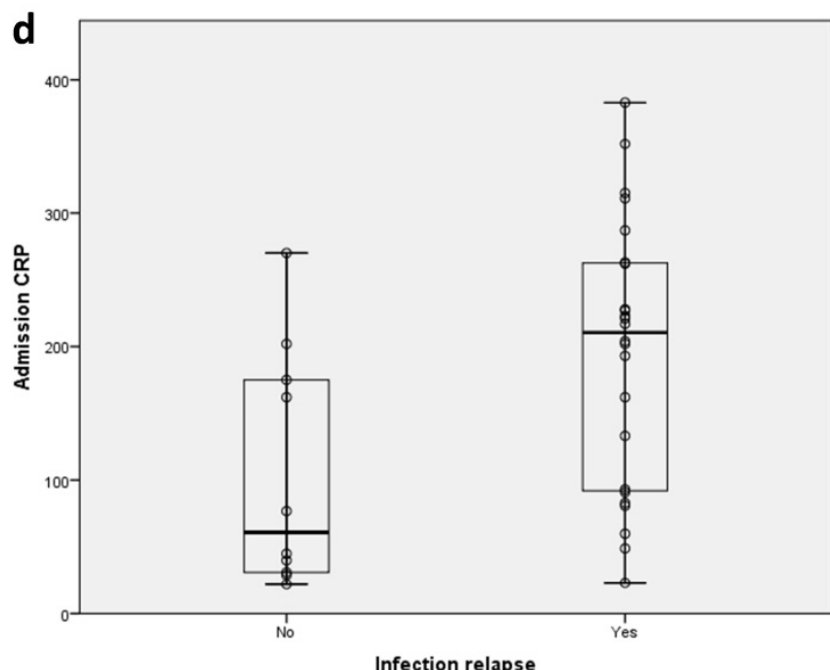

Figure 2. Clinical outcomes. a) Kaplan-Meier analysis of patient survival. b) Log rank analysis of patient survival between those with healed and non-healed pressure ulcers at time of final follow-up. c) Treatment failure. d) Admission C-reactive protein ( $\left.\mathrm{mg} \mathrm{L}^{-1}\right)$ at index presentation in the prediction of treatment failure.

\section{Microbiology}

Considering each index admission and any subsequent treatment failures as an infection episode, positive microbiology was available for $80 / 90$ episodes, with growth from a deep bone sample in 46 instances, blood culture in eleven, and a superficial wound swab alone in 26. Bacteraemia was due to $S$. aureus (4 MSSA, 1 MRSA), S. agalactiae $(\mathrm{n}=2), S$. dysgalactiae $(\mathrm{n}=1)$, E. faecium $(\mathrm{n}=1)$ and Gram negative bacilli (GNB; $n=3$; Table S3). Deep bone samples were obtained intra-operatively in $43 / 46$ episodes and by a Radiologist in 3 medically-managed episodes. In $13 / 18$ cases with sufficient data, antimicrobials were administered prior to surgery. Significant microbiology from deep bone samples is shown in Table S4. Infection was usually polymicrobial $(40 / 46)$ and microbiologically heterogeneous, with $S$. aureus, Enterococci, GNB and anaerobes the most frequent pathogens. Gram positive bacteria were involved in $43 / 46$, Gram negative in 26/46 and anaerobes in
16/46 (Figure S1). The sensitivity and specificity of superficial wound swabs for detection of $S$. aureus, Enterococci, GNB and anaerobes were calculated (Table 3), demonstrating inadequate performance when compared to deep bone samples from the same infection episode. In particular, the specificity of detection of S. aureus (36.4\%) and GNB (43.8\%) were particularly low. The sensitivity for detecting individual organisms from superficial swabs was also low: $47.4 \%$ for GNB, $50.0 \%$ for Enterococci, $55.6 \%$ for anaerobes and $76.5 \%$ for S. aureus. Overall, 45/91 isolates $(49.5 \%)$ from deep bone samples were detected on superficial swabs in episodes where both were obtained. 10/35 patients were colonised with MRSA and five developed MRSA infection.

Sequential intra-operative bone samples were available for 12 patients (median 2 infection episodes/patient, range 2-4) encompassing a total of 30 infection episodes a median of 84 days apart (IQR 23-371). All were polymicrobial. Considering the 
combined 101 isolates from these episodes, 44 (43.6\%) were involved in subsequent infections. Considering individual patients, in 9/12 individuals at least one organism was involved in a further infection episode.

\section{Discussion}

Pressure ulcer-related pelvic osteomyelitis is a complex infection but remains relatively understudied. In this retrospective cohort study of 35 patients we sought to ask clinically relevant questions. This study is limited by its small sample size, single-centre design, retrospective nature and an inability to determine the clinical characteristics determining decisions to manage patients with or without surgery (including patient preference to undergo surgery). If a patient relocated to outwith the study region then instances of treatment failure could have been missed by the case ascertainment strategy.

\section{Host nutritional status}

Anaemia and hypo-albuminaemia were identified as risk factors for a non-healing ulcer and could relate to host nutritional status in the context of a catabolic state induced by chronic inflammation. Pre-operative anaemia is associated with an increased risk of post-operative infections in patients undergoing orthopaedic surgery [11-13], with the effect thought to be associated with an increased requirement for post-operative blood transfusion [12-15]. Thus, there is a risk-benefit balance between transfusion-related immune modulation and optimising oxygenation of the hypo-perfused wound. Measures such as nutritional optimisation and haematinic assessment should be prioritised to reduce reliance on blood transfusion. It has been suggested that blood transfusion should be avoided intraoperatively [16] and, if anticipated, should be administered at least 48 hours before surgery.

Malnutrition is associated with multiple adverse outcomes following orthopaedic surgery: prolonged length of in-patient stay [17], delayed wound healing [18], surgical site infection (SSI) [19], sepsis [20], and death [20]. Serum albumin has been reported as a marker of nutritional status and hypo-albuminaemia $\left(<35 \mathrm{~g} \mathrm{~L}^{-1}\right)$ has consistently been associated with increased risk of SSI following orthopaedic surgery [17, 20-23]. A recent international consensus meeting on orthopaedic infections strongly agreed that malnutrition was a modifiable risk factor for delayed wound healing [24] and SSI [25]. A possible alternative explanation for the observed association with hypo-albuminaemia and ulcer healing is loss of albumin via wound exudate, in a process similar to that occurring in burns patients [26].
Table 1. Characteristics of included patients with pressure ulcer-related pelvic osteomyelitis $(n=35)$

\begin{tabular}{|c|c|}
\hline Characteristics & N (\%) \\
\hline Male (\%) & $26(74)$ \\
\hline Age, mean $(95 \% \mathrm{CI})$ years & $57.4(52.1-62.8)$ \\
\hline Neurological dysfunction & $32(91.4)$ \\
\hline Hemiplegia & $2(5.7)$ \\
\hline Paraplegia & $27(77.1)$ \\
\hline Quadriplegia & $3(8.6)$ \\
\hline \multicolumn{2}{|l|}{ Aetiology of neurologic dysfunction } \\
\hline Trauma & $10(28.6)$ \\
\hline Multiple sclerosis & $8(22.9)$ \\
\hline Congential & $7(20.0)$ \\
\hline Othera & $7(20.0)$ \\
\hline Defunctioning colostomy & 4 \\
\hline Long-term urinary catheter & 24 \\
\hline \multicolumn{2}{|l|}{ Cierny-Mader host classification } \\
\hline A & $8(22.9)$ \\
\hline B & $26(74.3)$ \\
\hline $\mathrm{C}$ & $1(2.9)$ \\
\hline \multicolumn{2}{|l|}{ Co-morbidities } \\
\hline Diabetes mellitus & $8(22.9)$ \\
\hline Ischaemic heart disease or heart failure & $6(17.1)$ \\
\hline Chronic lung disease & $4(11.4)$ \\
\hline Otherb & $4(11.4)$ \\
\hline \multicolumn{2}{|l|}{ Site of pressure ulcer } \\
\hline Ischium & 19 \\
\hline Sacrum & 13 \\
\hline Femur & 3 \\
\hline \multicolumn{2}{|l|}{ Site of osteomyelitis } \\
\hline Ischium & $18(51.4)$ \\
\hline Proximal femur & $5(14.3)$ \\
\hline Sacro-coccygeal & $5(14.3)$ \\
\hline Sacrum & $3(8.6)$ \\
\hline Ischium and pubic ramus & $2(5.7)$ \\
\hline Ischium and proximal femur & $1(2.9)$ \\
\hline Coccyx & $1(2.9)$ \\
\hline \multicolumn{2}{|l|}{ Diagnostic parameters at index admission } \\
\hline Total white cell count, $\times 10^{9} \mathrm{~L}^{-1}$ (mean, $\left.95 \% \mathrm{CI}\right)$ & $12.7(10.6-14.0)$ \\
\hline Neutrophil count, $\times 10^{9} \mathrm{~L}^{-1}($ mean, $95 \% \mathrm{CI})$ & $10.2(8.3-12.2)$ \\
\hline C-reactive protein, $\mathrm{mg} \mathrm{L}^{-1}$ (mean, $95 \% \mathrm{CI}$ ) & $168(132-205)$ \\
\hline \multicolumn{2}{|l|}{ Radiological findings $(n=33)^{c}$} \\
\hline Bone marrow oedema & $25(75.8)$ \\
\hline Bone cortex erosion & $19(57.6)$ \\
\hline Sequestra & $5(15.2)$ \\
\hline Myositis & $20(60.6)$ \\
\hline Collection or abscess & $15(45.5)$ \\
\hline \multicolumn{2}{|l|}{ Histopathological findings $(n=9)$} \\
\hline Acute (neutrophilic) inflammation & $1(11.1)$ \\
\hline Chronic (monocytic) inflammation & $4(44.4)$ \\
\hline Acute and chronic inflammation & $2(22.2)$ \\
\hline Reactive changes only & $2(22.2)$ \\
\hline Significant microbiology from deep bone sample $(n=25)$ & $25(100.0)$ \\
\hline Surgical intervention & $24(68.6)$ \\
\hline Debridement of tissue & $2(9.1)^{d}$ \\
\hline Debridement of tissue and bone & $20(90.9)^{\mathrm{d}}$ \\
\hline Soft tissue coverage (direct primary closure or flap) & $18(81.8)^{\mathrm{d}}$ \\
\hline \multicolumn{2}{|l|}{ Outcomes } \\
\hline Treatment failure & $25(71.4)$ \\
\hline Ulcer healinge & $12(36.4)^{\mathrm{e}}$ \\
\hline 1-year mortality & $8(22.9)$ \\
\hline \multicolumn{2}{|c|}{$\begin{array}{l}\text { a encephalitis }(n=3) \text {, subarachnoid haemorrhage }(n=1) \text {, stroke }(n=1) \text {, cauda equin } \\
\text { syndrome }(n=1) \text {, spinal cord infarct }(n=1) \text {. b Other: chronic kidney disease }(n=2) \text {; } \\
\text { chronic liver disease }(n=1) \text {; active malignancy }(n=1) \text {. c radiology results available } \\
\text { for } 33 / 35 \text { patients: } 26 \text { MRI, } 7 \text { CT.d data available for } 22 \text { patients.e at time of chart } \\
\text { review, data available for } 33 \text { patients. } f \text { relative to date of index admission. }\end{array}$} \\
\hline
\end{tabular}


Table 2. Receiver operator characteristic analysis of risk factors associated with outcomes

\begin{tabular}{|c|c|c|c|c|c|c|c|}
\hline & Threshold $^{a}$ & Sensitivity $(\%)$ & Specificity $(\%)$ & AUC & Rating & p-value & AUC 95\% CI \\
\hline \multicolumn{8}{|l|}{ Ulcer healing } \\
\hline Age at index admission & 59.5 years & 70 & 75 & 0.75 & Fair & 0.019 & $0.57-0.93$ \\
\hline Diabetes mellitus & NA & & & 0.66 & Poor & 0.144 & $0.47-0.85$ \\
\hline Admission haemoglobin & $111 \mathrm{~g} \mathrm{~L}^{-1}$ & 100 & 75 & 0.81 & Good & 0.039 & $0.63-0.99$ \\
\hline Admission albumin & $25 \mathrm{~g} \mathrm{~L}^{-1}$ & 80 & 81 & 0.84 & Good & 0.026 & $0.66-1.00$ \\
\hline Soft tissue coverage after debridement & NA & & & 0.73 & Fair & 0.032 & $0.55-0.92$ \\
\hline \multicolumn{8}{|l|}{ Treatment failure } \\
\hline No formal care support & NA & & & 0.72 & Fair & 0.045 & $0.53-0.91$ \\
\hline Post-traumatic neurological deficit & NA & & & 0.70 & Fair & 0.068 & $0.53-0.87$ \\
\hline Admission C-reactive protein & $184 \mathrm{mg} \mathrm{L}^{-1}$ & 63 & 80 & 0.78 & Fair & 0.013 & $0.60-0.95$ \\
\hline
\end{tabular}

a Threshold for optimal diagnostic accuracy derived by calculation of the Youden J statistic. NA: not applicable (categorical variable); AUC: area under the curve

Table 3. Sensitivity and specificity of superficial wound swabs compared to deep bone samples

\begin{tabular}{lll}
\hline & Sensitivity $\mathbf{( 9 5 \% ~ C I )}$ & Specificity (95\% CI) \\
\hline Staphylococcus aureus & $76.5(50.1-93.2)$ & $36.4(10.9-69.2)$ \\
Enterococci & $50.0(21.1-78.9)$ & $86.7(59.5-98.3)$ \\
Gram negative bacilli & $47.4(24.5-71.1)$ & $43.8(19.8-70.1)$ \\
Anaerobes & $55.6(21.2-86.3)$ & $84.2(60.4-96.6)$ \\
\hline
\end{tabular}

\section{Wound coverage}

Attempted soft tissue coverage was associated with eventual ulcer healing in this cohort. Two centres have reported good long-term outcomes using myo-cutaneous flaps. A flap was constructed in $157 / 157$ cases in one cohort (all pressure ulcers; 108 with underlying osteomyelitis) and 30/61 in another (all pelvic osteomyelitis; 41 with pressure ulcers) [10, 27]. In this latter cohort, $5 / 7$ recurrences occurred in patients with osteomyelitis due to pressure ulcers. One retrospective study specifically sought risk factors for ulcer recurrence after debridement, reporting a hazard ratio of 0.8 for flap use, but with a wide $95 \%$ confidence interval of 0.2 to 3.1 [28]. However, flaps were only constructed in 25/70 patients and underlying osteomyelitis was absent in 18/70 lesions. Another centre reported flap construction after debridement in only $7 / 55$ cases but no specific associated outcomes [29].

\section{Predicting and preventing treatment failure}

Treatment failure was predicted by admission CRP, lack of formal care support after discharge and a post-traumatic neurological deficit. CRP concentration at presentation has previously been found to independently predict treatment failure in musculoskeletal infections, possibly related to the burden of infection [30, 31].

In the context of preventing treatment failure, a holistic perspective is warranted, considering patient concordance with treatment, wound care and pressure off-loading. Any underlying psychosocial factors that contributed to initial ulcer development should be identified and addressed, including formal care support after hospital discharge [10, 32]. Whilst
Tissue Viability nursing input is the standard of care for patients in hospital, it is not known what wound care was delivered to patients in this cohort in the community after discharge.

A multi-disciplinary team approach to musculoskeletal infection links clinicians (infection specialists, musculoskeletal radiologists, anaesthetists, plastic surgeons and orthopaedic surgeons) and allied health and care professionals (nurses, dieticians, physiotherapists, occupational therapists, pharmacists, psychologists and social care co-ordinators) [33]. The cohort study from Dudareva and colleagues from the Oxford Bone Infection Unit reported a low recurrence rate $(11 \%)$ in the setting of an established multi-disciplinary approach to pelvic osteomyelitis [10]. The definition of treatment failure used in this study did not include re-admission for intravenous antimicrobials which we did include, and not all patients had pressure ulcer-related osteomyelitis. Therefore, outcomes are not directly comparable between our cohort and theirs.

\section{Microbiological sampling and aetiology}

Based on the results of deep bone cultures we report a microbial aetiology comparable to other investigators [6, 7, 10, 29]. Importantly, we show superficial wound swabs are poor predictors of deep bone microbiology, thus are uninformative in determining aetiology of underlying osteomyelitis. One series reported that the implicated pathogen identified during recurrent episodes was unrelated to the index isolate in $86 \%$ of cases, and our data similarly show that only $43.6 \%$ of recovered organisms were identified in subsequent infections [28]. Therefore, prior microbiology results do not reliably predict the aetiology of subsequent infection episodes and there is evidence for microbiological recurrence and de novo re-infection occurring simultaneously.

\section{Antimicrobial management}

We identified significant heterogeneity in the total duration of antimicrobial therapy during the index admission but no association with subsequent 
treatment failure. To our knowledge this is the first report of this finding in a cohort of patients exclusively with pressure ulcer-related pelvic osteomyelitis, and supports similar findings by other investigators who have demonstrated that antimicrobial duration is not associated with healing of infected sacral pressure ulcers (with or without underlying osteomyelitis) [6, 34, 35]. The recent OVIVA trial included $6.8 \%$ patients with spinal/pelvic osteomyelitis but overall 322/376 patients with chronic osteomyelitis (any site) had undergone debridement, therefore to extrapolate the trial findings to pressure ulcer-related pelvic osteomyelitis, further work will be required to define the sub-group of patients requiring surgical intervention [36]. The large cohort study from Bodavula and colleagues reports that combined medical and surgical management is associated with less re-admissions in the following year compared to antimicrobials alone [29]. Whilst this is an informative finding, the chronicity of this infection dictates that analysis of more than one year of follow-up will be required to reach definitive conclusions. Finally, the extent of osteomyelitis is likely to be a relevant factor when determining the length of antimicrobial therapy required, for example superficial cortical versus medullary involvement. There was insufficient histological information available to comment on this in our cohort.

\section{Anticipatory care planning}

In this series, $43 \%$ of patients had died after mean follow-up of 3.7 years. Other investigators have reported $20-25 \%$ mortality with mean follow-up of 2.8-4.7 years [10, 37]. These findings speak to the importance of anticipatory care planning and 'Realistic Medicine' following an index admission with pressure-ulcer related pelvic osteomyelitis [38]. Clinicians must continuously evaluate the appropriateness of aggressive management, entailing deep bone sampling, surgical debridement and embarking on lengthy and complex antimicrobial regimens. Such measures should not be pursued if and when a time arises when any benefits derived are outweighed by deleterious effects on the patient's overall physiological, psychological and social wellbeing, viewed from the patient's perspective. This is particularly pertinent when considering the high treatment failure rate resulting in re-admission and underscores the importance of preventing this. Realistic goals of care could include promoting healing of existing ulcers, preventing additional ulcers and treating acute flares of SSTI involving the ulcer with brief courses of antimicrobials.

\section{Conclusions}

Identification of the sub-group of patients who will benefit from surgery remains an important goal to advance the management of this disease. Formal personal care and nutrition are potentially modifiable factors associated with outcomes and should feature in strategies to optimise surgical outcomes. Admission CRP could be used to identify patients at risk of poor outcomes who may need closer followup. For patients undergoing surgical intervention, this should be performed as a combined ortho-plastic procedure to facilitate adequate debridement and early soft tissue reconstruction, when primary closure is not possible [10, 27]. Microbiological evaluation should involve deep bone sampling and not rely on superficial wound swabs. Antimicrobial duration is not associated with risk of treatment failure and shorter courses may be appropriate in some cases. The role of antimicrobials may be secondary to optimising the bio-psycho-social factors required for successful surgical intervention and subsequent wound healing. Almost one quarter of patients died within one-year of their index episode and therefore anticipatory care planning should be considered. A holistic approach to management is required, including dietetics, careful discharge planning, social care, tissue viability, infection specialists, plastic surgeons and orthopaedic surgeons.

\section{Supplementary Material}

Supplementary tables.

http://www.jbji.net/v05p0067s1.pdf

\section{Acknowledgements}

We gratefully acknowledge the assistance of Jason Clark at NHS Lothian Analytical Services for running the EPR search to identify possible cases.

\section{Author Contributions}

RKS conceived the research questions. AHRWS maintained a database of surgically managed cases. CDR and STJT collected and analysed the data. RKS and AHRWS supervised the work. All authors contributed to manuscript preparation.

\section{Competing Interests}

The authors have declared that no competing interest exists.

\section{References}

1. Cunha BA. Osteomyelitis in elderly patients. Clin Infect Dis. 2002; 35: 287-93.

2. Han H, Lewis VL, Jr., Wiedrich TA, Patel PK. The value of Jamshidi core needle bone biopsy in predicting postoperative osteomyelitis in grade IV pressure ulcer patients. Plast Reconstr Surg. 2002; 110: 118-22.

3. Wong D, Holtom P, Spellberg B. Osteomyelitis Complicating Sacral Pressure Ulcers: Whether or Not to Treat With Antibiotic Therapy. Clin Infect Dis. 2019; 68: $338-42$. 
4. Turk EE, Tsokos M, Delling G. Autopsy-based assessment of extent and type of osteomyelitis in advanced-grade sacral decubitus ulcers: a histopathologic study. Arch Pathol Lab Med. 2003; 127: 1599-602.

5. Darouiche RO, Landon GC, Klima M, Musher DM, Markowski J. Osteomyelitis associated with pressure sores. Arch Intern Med. 1994; 154: 753-8.

6. Sugarman B, Hawes S, Musher DM, Klima M, Young EJ, Pircher F. Osteomyelitis beneath pressure sores. Arch Intern Med. 1983; 143: 683-8

7. Brunel AS, Lamy B, Cyteval C, Perrochia H, Teot L, Masson R, et al. Diagnosing pelvic osteomyelitis beneath pressure ulcers in spinal cord injured patients: a prospective study. Clin Microbiol Infect. 2016; 22: 267.e1-8.

8. Larson DL, Gilstrap J, Simonelic K, Carrera GF. Is there a simple, definitive, and cost-effective way to diagnose osteomyelitis in the pressure ulcer patient? Plast Reconstr Surg. 2011; 127: 670-6.

9. Lewis VL, Jr., Bailey MH, Pulawski G, Kind G, Bashioum RW, Hendrix RW. The diagnosis of osteomyelitis in patients with pressure sores. Plast Reconstr Surg. 1988; 81: 229-32.

10. Dudareva M, Ferguson J, Riley N, Stubbs D, Atkins B, McNally M. Osteomyelitis of the Pelvic Bones: A Multidisciplinary Approach to Treatment. J Bone Jt Infect. 2017; 2: 184-93.

11. Greenky M, Gandhi K, Pulido L, Restrepo C, Parvizi J. Preoperative anemia in total joint arthroplasty: is it associated with periprosthetic joint infection? Clin Orthop Relat Res. 2012; 470: 2695-701.

12. Myers E, Grady PO, Dolan AM. The influence of preclinical anaemia on outcome following total hip replacement. Arch Orthop Trauma Surg. 2004; 124: 699-701.

13. Morales CH, Escobar RM, Villegas MI, Castaño A, Trujillo J. Surgical site infection in abdominal trauma patients: risk prediction and performance of the NNIS and SENIC indexes. Can J Surg. 2011; 54: 17-24

14. Steinitz D, Harvey EJ, Leighton RK, Petrie DP. Is homologous blood transfusion a risk factor for infection after hip replacement? Can J Surg. 2001; 44: $355-8$

15. Alexander JW, Solomkin JS, Edwards MJ. Updated recommendations for control of surgical site infections. Ann Surg. 2011; 253: 1082-93.

16. Kendall SJ, Weir J, Aspinall R, Henderson D, Rosson J. Erythrocyte transfusion causes immunosuppression after total hip replacement. Clin Orthop Relat Res. 2000: $145-55$.

17. Lavernia CJ, Sierra RJ, Baerga L. Nutritional Parameters and Short Term Outcome in Arthroplasty. J Am Coll Nutr. 1999; 18: 274-8.

18. Gherini S, Vaughn BK, Lombardi AV, Mallory TH. Delayed wound healing and nutritional deficiencies after total hip arthroplasty. Clin Orthop Relat Res. 1993; 293: 188-95.

19. Cross MB, Yi PH, Thomas CF, Garcia Ja. Evaluation of Malnutrition in Orthopaedic Surgery. J Am Acad Orthop Surg. 2014; 22: 193-9.

20. McLaren AC, Lundy DW. AAOS Systematic Literature Review: Summary on the Management of Surgical Site Infections. J Am Acad Orthop Surg. 2018; 27: e717-20.

21. Del Savio GC, Zelicof SB, Wexler LM, Byrne DW, Reddy PD, Fish D, et al. Preoperative nutritional status and outcome of elective total hip replacement. Clin Orthop Relat Res. 1996; 326: 153-61.

22. Yuwen $\mathrm{P}$, Chen $\mathrm{W}$, Lv H, Feng C, Li Y, Zhang T, et al. Albumin and surgical site infection risk in orthopaedics: a meta-analysis. BMC Surg. 2017; 17: 7.

23. Zainul-Abidin S, Amanatullah DF, Anderson MB, Austin M, Barretto JM, Battenberg A, et al. General Assembly, Prevention, Host Related General: Proceedings of International Consensus on Orthopedic Infections. J Arthroplasty. 2019; 34: s13-35.

24. Chotanaphuti T, Courtney PM, Fram B, In den Kleef NJ, Kim TK, Kuo FC, et al. Hip and Knee Section, Treatment, Algorithm: Proceedings of International Consensus on Orthopedic Infections. J Arthroplasty. 2019; 34: s393-7.

25. Azboy I, Bedair H, Demirtas A, Ford E, Gahramanov A, Klement MR, et al. General Assembly, Prevention, Risk Mitigation, General Factors: Proceedings of International Consensus on Orthopedic Infections. J Arthroplasty. 2019; 34: s55-9.

26. Prez-Guisado J, de Haro-Padilla JM, Rioja LF, Derosier LC, de la Torre JI. Serum albumin levels in burn people are associated to the total body surface burned and the length of hospital stay but not to the initiation of the oral/enteral nutrition. Int J Burns Trauma. 2013; 3: 159-63.

27. Marriott R, Rubayi S. Successful Truncated Osteomyelitis Treatment for Chronic Osteomyelitis Secondary to Pressure Ulcers in Spinal Cord Injury Patients. Ann Plast Surg. 2008; 61: 425-9.

28. Jugun K, Richard J-C, Lipsky BA, Kressmann B, Pittet-Cuenod B, Suv. Factors Associated With Treatment Failure of Infected Pressure Sores. Ann Surg. 2016; 264: 399-403.

29. Bodavula P, Liang SY, Wu J, VanTassell P, Marschall J. Pressure Ulcer-Related Pelvic Osteomyelitis: A Neglected Disease? Open Forum Infect Dis. 2015; 2: 112.

30. Wouthuyzen-Bakker M, Sebillotte M, Lomas J, Taylor A, Palomares EB, Murillo $\mathrm{O}$, et al. Clinical outcome and risk factors for failure in late acute prosthetic joint infections treated with debridement and implant retention. J Infect. 2019; 78: 40-7.

31. Markanday A. Acute Phase Reactants in Infections: Evidence-Based Review and a Guide for Clinicians. Open Forum Infect Dis. 2015; 2: 98

32. Wong D, Holtom $P$, Spellberg B. Osteomyelitis complicating sacral pressure ulcers: whether or not to treat with antibiotic therapy. Clin Infect Dis. 2018; 68: $338-42$.
33. Yan $\mathrm{CH}$, Arciola $\mathrm{CR}$, Soriano A, Levin LS, Bauer TW, Parvizi J. Team Approach: The Management of Infection After Total Knee Replacement. JBJS Rev. 2018; 6: e9.

34. Jugun K, Richard JC, Lipsky BA, Kressmann B, Pittet-Cuenod B, Suva D, et al. Factors Associated With Treatment Failure of Infected Pressure Sores. Ann Surg. 2016; 264: 399-403.

35. Firriolo JM, Ganske IM, Pike CM, Caillouette C, Faulkner HR, Upton J, 3rd, et al. Long-term Outcomes After Flap Reconstruction in Pediatric Pressure Ulcers. Ann Plast Surg. 2018; 80: 159-63.

36. Li HK, Rombach I, Zambellas R, Walker AS, McNally MA, Atkins BL, et al. Oral versus Intravenous Antibiotics for Bone and Joint Infection. $\mathrm{N}$ Engl J Med. 2019; 380: 425-36.

37. Ramesh R, Gaston MS, Simpson AHRW. Chronic osteomyelitis of the pelvis. Acta Orthop Belg. 2013; 79: 280-6.

38. Care DoHaS. Scottish Government. Realising Realistic Medicine-Chief Medical Officer's annual report 2015-16. 2016. 